\title{
Radiative nucleon capture - a dual approach
}

\author{
E. Běták ${ }^{1,2, a}$, A. Likar $^{3,4}$, and T. Vidmar ${ }^{3}$ \\ 1 Institute of Physics SAS, 84511 Bratislava, Slovakia \\ 2 Silesian University, 74601 Opava, Czech Republic \\ 3 Institute "J. Stefan", 1001 Ljubljana, Slovenia \\ ${ }^{4}$ University of Ljubljana, 1000 Ljubljana, Slovenia
}

\begin{abstract}
The nucleon radiative capture reactions represent a great interest for possible new ways of production of therapeutic and diagnostic isotopes and also for their deep insight into the details of nuclear reaction process itself. The high-energy gammas originating from the reactions of nucleon radiative capture at incident energies close to or above $10 \mathrm{MeV}$ can be described by either the semi-direct-direct model or within the pre-equilibrium approach. The detailed view on the origin of the optical model potential indicates the origin of its imaginary part. Both approaches are compared together and also to the data.
\end{abstract}

\section{Introduction}

Reactions of radiative capture of nucleons above the resonance region - even though very difficult to be measured due to their low cross sections - serve as a challenge for different $\gamma$ emission mechanisms already for decades. There are two competing and complementary models to describe the $\gamma$ emission in the continuum region, namely the direct-semi-direct model (DSD) and the pre-equilibrium decay.

The first one (see [1-3]) has proved its excellent capabilities in describing the transitions going directly to discrete states with well specified quantum numbers (i.e., those strongly of single-particle nature, usually very close to the (doubly-) magic nuclei or at least near closed shells). The states are described by the use of the optical model wave functions.

The pre-equilibrium model (see, e.g., [4]), on the other hand, is a statistical approach to the problem in its nature. Anyway, it appeared to be rather successful to describe the $\gamma$ energy spectra in the continuum region in $14 \mathrm{MeV}$ neutroninduced reactions [5-7]. The study of excitation functions extended the applicability of the model to energies from few $\mathrm{MeV}$ to about $30 \mathrm{MeV}$. Significant improvement was achieved by the incorporation of spin into the formalism of the pre-equilibrium exciton model [8,9], which enabled also pre-equilibrium calculations leading to discrete states and the comparison to the direct-semi-direct model calculations.

Both models have some common features, even though they are strictly complementary in their underlying physics. Whereas the direct-semi-direct model deals with wave functions and specific interactions of nucleons, while completely ignoring competing processes, the pre-equilibrium models are of statistical nature and they deeply involve quantities like the level densities, etc. The competition - e.g., of the nucleon emission - is naturally contained there. What is common to both models is that they are both able to reproduce (more-orless) the data corresponding to hard $\gamma$ emission observed in radiative nucleon capture at energies above few MeV [10].

${ }^{a}$ Presenting author, e-mail: betak@savba.sk
The available experimental data are not frequent due to low cross sections and - at the same time - low detector efficiency for these hard $\gamma$ 's.

The reactions of nucleon radiative capture are also a suitable tool for their deep insight into the details of nuclear reaction process itself. The latter can be studied in more detail by reactions leading to discrete and/or semi-discrete states; more frequently those induced by protons are studied (due to the energy resolution).

\section{Direct-semi-direct model}

DSD is formally a microscopic approach and the wave functions of nuclei (i.e., those strongly of single-particle nature, usually very close to the (doubly-) magic nuclei or at least near closed shells) and of the incident nucleon with well defined quantum numbers are necessary to be known. The interaction is described by the use of the optical model. In doing that, one usually ignores the coupling of discrete states involved in the reactions to those in continuum. The price for this neglecting is the appearance of non-Hermitian effective Hamiltonians, as recently pointed out by Likar and Vidmar [11].

Reference [11] has shown - though only within a toy model - that the imaginary part of the (effective) optical model potential arises from the neglect of the coupling of the "participant" state to other(s) (continuum) excited state(s). Similar coupling has been identified several decades ago in atomic physics by Fano [12]. Below, we shall shortly summarize the basic ideas of ref. [11].

If we consider the ground state $|0\rangle$ and the excited states $\left|\psi_{i}\right\rangle$, which are the eigen states of the Hamiltonian, the total wave function can be written

$$
|\Psi\rangle=\alpha(x)|0\rangle+\sum_{i} \beta_{i}(x)\left|\psi_{i}\right\rangle,
$$

where $\alpha$ and $\beta_{i}$ 's are the particle parts of the wave functions, and $x$ stands for all their quantum numbers including energy ${ }^{1}$.

\footnotetext{
${ }^{1}$ We shall omit writing $x$ in the formulae below in the cases where it does not cause any confusion.
} 
Let $V_{i}$ be the interaction part of the Hamiltonian, which couples the ground state to the $i$-th excited one, $\left\langle\psi_{i}|H| 0\right\rangle$. The particle parts $\alpha$ and $\beta_{i}$ 's are coupled together

$$
\begin{array}{r}
\alpha^{2}(x)=\left[1+\sum_{i} \frac{\left|V_{i}\right|^{2}}{E_{i}-E}\right]^{-1} \\
\beta_{i}(x)=-\frac{V_{i}}{E_{0}-E} \alpha(x) .
\end{array}
$$

As we are interested in the processes involving the ground state, one can eliminate $\beta$ 's from the above equations, which leads to

$$
\left(E-E_{0}\right) \alpha=\sum_{i} \frac{\alpha\left|V_{i}\right|^{2}}{E-E_{i}},
$$

and after introducing the energy in the complex plane, substituting $E$ by $E^{+}=E+i \eta$ and replacing the summation by an integration, one arrives to the Breit-Wigner formula with energy $E=E_{0}-i \Gamma / 2$, where the width is $\Gamma=\frac{2 \pi}{D}|V(E)|^{2}$. As a consequence of ignoring the continuum, the effective potential becomes complex.

If we keep all the states in considerations, we can equally arrive to the Breit-Wigner formula, but without the need to use the complex potential. Thus, the imaginary part is the price neglecting the other states.

The basic idea was illustrated on a case study of a system (nucleus) having just two states, the ground state and the first excited one, denoted for simplicity $|0\rangle$ and $|1\rangle$, respectively [11]. Further on, let us assume that $V$ is just a zero-range interaction

$$
V=\mathcal{V}(x)\left(a^{\dagger}+a\right)=g \delta(x)\left(a^{\dagger}+a\right),
$$

where $a^{\dagger}$ is the operator switching nucleus from $|0\rangle$ to the $|1\rangle$ state and $a$ the opposite one.

From this toy model we arrive to the conclusions that

- The wave function of the ground state is not only $\alpha|0\rangle$, but it has its $|1\rangle$ component as well. The full transition amplitude of the radiative nucleon capture from the initial state $\left|\Psi_{i}\right\rangle$ to the final ground state $\left|\Psi_{g}\right\rangle$ is $T_{\gamma}=\left\langle\Psi_{i}\left|H_{\gamma}\right| \Psi_{g}\right\rangle=$ $\int \alpha^{*} H_{\gamma} \alpha_{g} \mathrm{~d} x+\int \beta^{*} H_{\gamma} \beta_{g} \mathrm{~d} x$. Usually, only the first term is taken into account.

- After using the specific zero-range interaction, the transition amplitude is $T_{\gamma}=\int \alpha^{*}\left[H_{\gamma}+g^{2} G_{\beta}^{*(+)} H_{\gamma} G_{g}\right] \alpha_{g} \mathrm{~d} x$. Here, $G^{+}$is the Green function for the scattering state and $G_{g}$ that for the ground one. Thus, we get the operator of the electromagnetic transition $H_{\gamma}$ dressed to become an effective operator $\mathcal{H}_{\gamma}=H_{\gamma}\left(1+g^{2} G_{\beta}^{*(+)} G_{g}\right)$.

In the above, the derived behaviour is, we believe, of a general nature. The specific formulae given above have been, however, derived just within the simplified toy model [11].

\section{Pre-equilibrium description and calculations}

We used the computer codes EMPIRE-II v. 2.19 [13] and TALYS [14]. At excitation energies above $10 \mathrm{MeV}$, both are based on the pre-equilibrium single-particle radiative mechanism $[5,6]$, which has proved to be very successful at the incident energies below about $30 \mathrm{MeV}$ in previous codes (see, e.g., refs. [15,16]). On the other hand, it gives also a good and reliable description at energies as low as about $5 \mathrm{MeV}[17,18]$. The $\gamma$ emission rates can be expressed as $[5,6]$

$$
\lambda_{\gamma}\left(n, E, \varepsilon_{\gamma}\right)=\frac{\varepsilon_{\gamma}^{2} \sigma_{\mathrm{GDR}}\left(\varepsilon_{\gamma}\right)}{\pi^{2} \hbar^{3} c^{2}} \frac{\sum_{m=n, n-2} b\left(m, \varepsilon_{\gamma}\right) \omega\left(m, E-\varepsilon_{\gamma}\right)}{\omega(n, E)},
$$

with $b$ 's the corresponding branching ratios (see [5,6]) and $\omega$ 's the corresponding state densities with specified energy and the number of excitons. With the inclusion of spin, however, the branching ratios become much more complicated, but fortunately - they factorize [8], and the energy-dependent parts are identical to those of the spin-independent ones.

The photo-absorption cross section $\sigma_{\mathrm{GDR}}$ is usually taken in the form of the giant dipole resonance approximated by the corresponding Lorentzian (or a double-humped Lorentzian in the case of deformed nuclei).

A counterpart to the non-participating states influencing the (effective) nuclear potential can be found also in the preequilibrium statistical picture of the single-particle radiative mechanism. The justification of equation (5) stems from the assumption of the $1 \mathrm{p} 1 \mathrm{~h}$ GDR excitation. The model description of a $\gamma$ capture on an even-even target leads to the particlehole pair creation. However, the $\gamma$ capture as well as its emission is (are) associated with two different processes the other one leaves the exciton number unchanged and just shifts a particle (or a hole) in energy. This is the origin of the branching ratios in (5) and incorporation of not-involved states in the detailed balance was just the difference between the original [5] and the improved [6] formulation of the preequilibrium gamma decay. The justification of this inclusion given in [6] was based on the equilibrium limit and different interpretation of the Brink-Axel hypothesis, so that the present argumentation, coming from the behaviour of the effective optical potential, gives an additional support for that.

There are some differences between TALYS and EMPIRE which are important for the pre-equilibrium stage of the reaction. Apart of using not identical libraries for different parameters and allowing their users for different advanced options, probably the most significant one is that the basic approach is a two-component one (i.e., distinguishing between the neutrons and the protons) in TALYS, whereas one-component formulation with a charge factor is used in EMPIRE $^{2}$.

Previous calculations done using other codes demonstrated the sensitivity of the calculations to the details of the level density parameters. Thus, the results can differ by as much as one order of magnitude. Also the influence of the temperaturedependent width of the GDR was clearly demonstrated, which is rather surprising at excitation energies well below $50 \mathrm{MeV}$ [17]; the detailed form of the GDR and the differences among different level density models are only of marginal influence and practically may be neglected $[22,23]$. Thus, the calculations are relatively reliable far off the closed shells and

\footnotetext{
2 More discussion on the differences of these two codes is in ref. [21].
} 


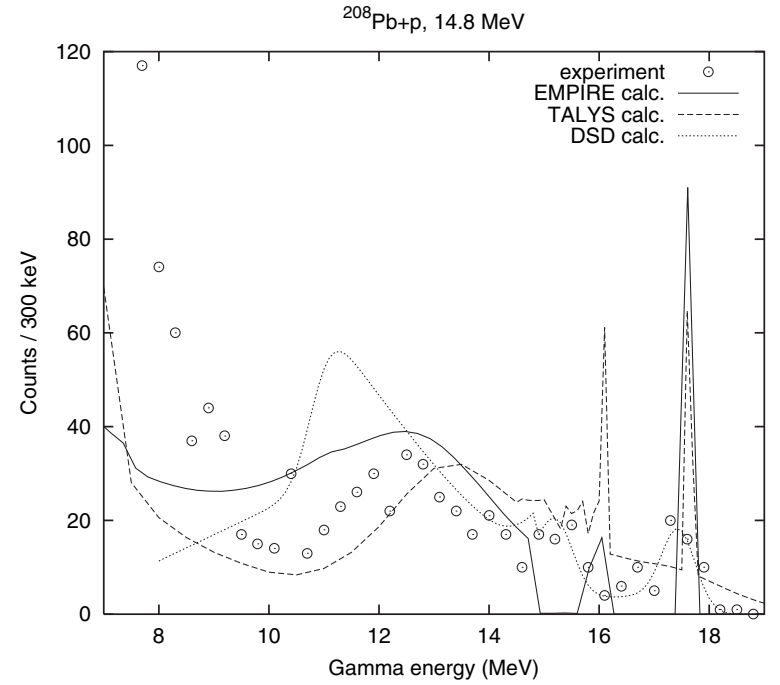

Fig. 1. The $\gamma$-spectrum measured in coincidence with the $f_{7 / 2}$ $(896 \mathrm{keV})$ transition in ${ }^{208} \mathrm{~Pb}+\mathrm{p}$ at $14.8 \mathrm{MeV}$ [20] compared with theoretical predictions.

(simultaneously) close to the beta-stability line, and rather unsure near closed (or even doubly closed) one(s), where one has to pay the upmost care to the parameters (see, e.g., [17]). Generally, there is no straightforward solution for nuclei close to the drip lines, and calculations performed with different model assumptions and/or codes may serve as a rough estimate how reliable the evaluation may be.

\section{Gamma spectra in proton radiative capture above $10 \mathrm{MeV}$}

Recently, Lipoglavšek et al. published detailed measurements of the ${ }^{208} \mathrm{~Pb}(\mathrm{p}, \gamma)$ reaction at incident energies between 8 and $19 \mathrm{MeV}[19,20]$. They presented the excitation functions of discrete states of ${ }^{209} \mathrm{Bi}$ populated in this reaction, and also the $\gamma$ spectra for several incident energies. We have taken these measurements as a basis for our calculations. For the statistical calculations (the codes TALYS and EMPIRE contain as their essential parts the pre-equilibrium emission), the application of the models to reactions near the closed shells is not straightforward, and a special attention should be paid to different quantities, especially the level densities. This has been already demonstrated in our previous papers, where also a special care had to be applied for the reactions on double-closed ${ }^{208} \mathrm{~Pb}[17$, $24]$. For the DSD calculations, on the other hand, the doubleclosed nucleus is an advantage, as the single-particle states with well defined wave functions appear more clearly here.

We have applied both approaches, the DSD model and the pre-equilibrium decay, to the reactions of proton radiative capture to discrete states in ${ }^{208} \mathrm{~Pb}(\mathrm{p}, \gamma)$. As an example, the $\gamma$ energy spectra obtained at 14.8 and $16.9 \mathrm{MeV}$ in coincidence with the $896 \mathrm{keV} f_{7 / 2^{-}} \rightarrow$ g.s. transition are presented in figures 1 and $2 .^{3}$ The overall agreement of the three

\footnotetext{
${ }^{3}$ Preliminary calculations of the pre-equilibrium $\gamma$ spectra from this reaction and also the excitation functions to the discrete states
}

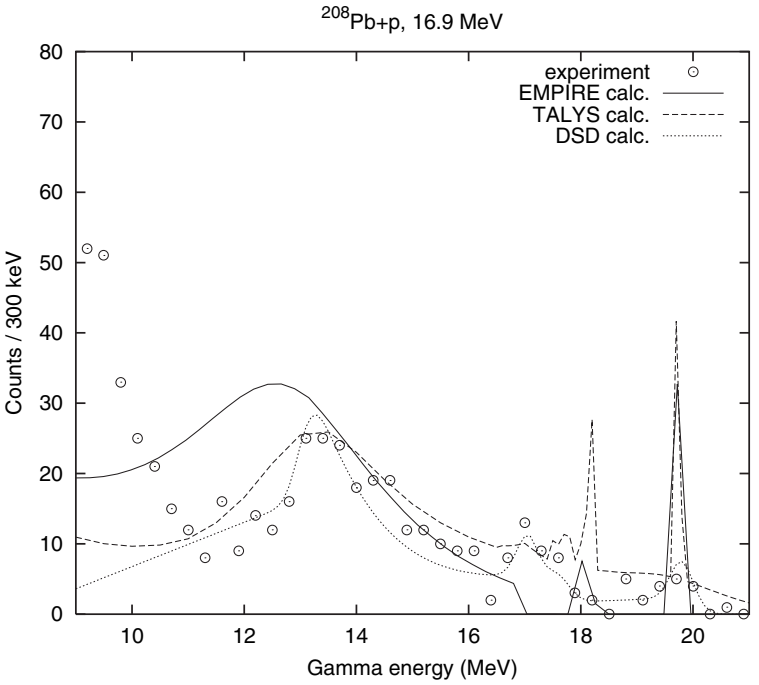

Fig. 2. Same as figure 1, but at $16.9 \mathrm{MeV}$.

calculations and the data is reasonable. However, some differences can still be marked (e.g., the peaks corresponding to direct capture to the low-lying discrete states are much more pronounced in the pre-equilibrium calculations than in the DSD ones or in the experimental data). In fact, direct $\gamma$ transitions to discrete excited states have been limited to the states of excitation energy below $2.7 \mathrm{MeV}$ (EMPIRE) or 3.2 MeV (TALYS) in our calculations.

The experimental data are given in counts, whereas the calculations by TALYS and EMPIRE give cross sections in absolute values. For the plots, the calculations have been scaled to come close to experimental data in the region of broad peak close to $13 \mathrm{MeV}$. In these calculations, the set of considered discrete states (together with their branching ratios) has been taken into account up to about $3 \mathrm{MeV}$ of excitation energy (2.7 MeV in EMPIRE and 3.2 MeV in TALYS), and above that energy only a continuous part has been considered.

In the comparison of the two complementary theoretical approaches, one should keep in mind that only the primary transitions are taken into account in the DSD approach, and all possible $\gamma$ cascades and $\gamma$-to-nucleon competitions are included in the other two. The absence of the cascades and of the competition of other open channels is responsible for very low low-energy tail in the calculated DSD $\gamma$ spectra. The experimental points, however, show high cross sections at low $\gamma$ energies, as the calculations by EMPIRE and TALYS also do.

\section{Conclusions}

We have pointed out some underlying features which lead to the appearance of the complex effective potential used in the

have been published in refs. [10,21]. The EMPIRE calculation is given without accounting for $\gamma$ 's after nucleon emission, for better comparison to the DSD calculations, The visible gap in the EMPIRE spectrum near $18 \mathrm{MeV}$ is just an artefact of insufficient dimension of the computer output table listing. 
optical model, and indicated its possible counterpart in the pre-equilibrium statistical model, in both cases applied to the nucleon radiative capture reactions at incident energies above $10 \mathrm{MeV}$. Both DSD and pre-equilibrium calculations have been compared to the data of the ${ }^{208} \mathrm{~Pb}(\mathrm{p}, \gamma)$ reactions from few $\mathrm{MeV}$ to about $20 \mathrm{MeV}$. Therein, the statistical way of pre-equilibrium description does not work so nicely - it is explained by specific non-statistical behaviour in the vicinity of doubly-magic ${ }^{208} \mathrm{~Pb}$. Two codes, EMPIRE and TALYS, used for the pre-equilibrium calculations give results of similar quality, and there is no clear dominance of one of them over the other.

This work has been supported in part by VEGA Grant No. 2/7115/27 and by the Slovene-Slovak collaboration project No. SK-SI-2006.

\section{References}

1. G.E. Brown, Nucl. Phys. 57, 339 (1964).

2. M. Potokar, Phys. Lett. B 46, 346 (1973).

3. A. Likar, T. Vidmar, Nucl. Phys. A 591, 458 (1995).

4. E. Gadioli, P. Hodgson, Pre-Equilibrium Nuclear Reactions (Clarendon Press, Oxford 1992).

5. E. Běták, J. Dobeš, Phys. Lett. B 84, 368 (1979).

6. J.M. Akkermans, H. Gruppelaar, Phys. Lett. B 157, 95 (1985).

7. F. Cvelbar, E. Běták, Z. Phys. A 332, 163 (1989).

8. P. Obložinský, Phys. Rev. C 35, 407 (1987).

9. P. Obložinský, M.B. Chadwick, Phys. Rev. C 41, 1652 (1990).

10. E. Běták, in Capture Gamma-Ray Spectroscopy and Related Topics: 12th International Symposium, Notre Dame, Indiana (USA), 4-9 Sept. 2005, edited by A. Woehr, A. Aprahamian. AIP Conference Proc., Vol. 819 (Amer. Inst. Phys., Melville, New York 2006), p. 226.
11. A. Likar, T. Vidmar, in em NEMEA-3 (Neutron Measurement, Evaluation and Application Enlargement Workshop), Borovec (Bulgaria), Oct. 2006 (to be published).

12. U. Fano, Phys. Rev. 124, 1866 (1961).

13. M. Herman, P. Obložinský, R. Capote, A. Trkov, V. Zerkin, M. Sin, B. Carlson, Report NNDC-BNL, Upton, USA, 2005.

14. A. Koning, S. Hilaire, M. Duijvestijn, Report 21297/04.62741/P FAI/AK/AK, NRG Petten, 2004.

15. E. Běták, Report INDC(CSR)-016/LJ, IAEA Vienna, 1989.

16. E. Běták, P. Obložinský, Report INDC(SLK)-001, IAEA Vienna, 1993.

17. E. Běták, F. Cvelbar, J. Kopecky, Phys. Rev. C 46, 945 (1992).

18. F. Cvelbar, E. Běták, A. Likar, J. Phys. G 21, 377 (1995).

19. M. Lipoglavšek, R. Bark, M. Benatar, E. Gueorguieva, J. Kau, F. Komati, P. Kwinana, J. Lawrie, G. Mabala, P. Maine, S. Mukhrejee, S. Mullins, S. Murray, N. Ncapayi, R. Newman, P. Vymers, A. Likar, M. Vencelj, T. Vidmar, Phys. Lett. B 593, 61 (2004).

20. A. Likar, M. Lipoglavšek, M. Vencelj, T. Vidmar, R. Bark, E. Gueorguieva, F. Komati, J. Lawrie, S. Maliage, S. Mullins, S. Murray, T. Ramashidzha, Phys. Rev. C 73, 044609 (2006).

21. E. Běták, A. Likar, M. Lipoglavšek, M. Vencelj, T. Vidmar, in 11th Internat. Conf. Nucl. Reacts. Mechanisms, Varenna, June 12-16, 2006, edited by E. Gadioli (Univ. Milano, Ric. Sci. Educ. Perm., Suppl. 126, Milano, 2006), p. 203.

22. Nuclear Data for the Production of Therapeutic Radionuclides. Summary Report of First Research Coordination Meeting. Prepared by J.-Ch. Sublet and R. Paviotti-Corcuera. Report INDC(NDS)-444, IAEA Vienna, 2003

23. Nuclear Data for the Production of Therapeutic Radionuclides. Summary Report of Second Research Coordination Meeting. Prepared by J.-Ch. Sublet and R. Capote Noy. Report INDC(NDS)-465, IAEA Vienna, 2004

24. E. Běták, F. Cvelbar, A. Likar, T. Vidmar, Nucl. Phys. A 686, 204 (2001). 